\title{
Le "modèle participatif" de développement rural
} est-il "alternatif" ?

Éléments pour une anthropologie de la culture des "développeurs"

Jean-Pierre Chauveau

\section{OpenEdition}

\section{Journals}

Édition électronique

URL : http://journals.openedition.org/apad/380

DOI : 10.4000/apad.380

ISSN : 1950-6929

Éditeur

LIT Verlag

Édition imprimée

Date de publication : 15 mars 1992

\section{Référence électronique}

Jean-Pierre Chauveau, « Le "modèle participatif" de développement rural est-il "alternatif" ? », Bulletin de I'APAD [En ligne], 3 | 1992, mis en ligne le 05 juillet 2006, consulté le 07 septembre 2020. URL : http:// journals.openedition.org/apad/380; DOI : https://doi.org/10.4000/apad.380

Ce document a été généré automatiquement le 7 septembre 2020

Bulletin de l'APAD 


\title{
Le "modèle participatif" de développement rural est-il "alternatif" ?
}

\author{
Éléments pour une anthropologie de la culture des "développeurs"
}

\author{
Jean-Pierre Chauveau
}

Dans un article sur "la place de la Participation dans le développement rural" (Cohen and Uphoff 1980), deux spécialistes américains des aspects institutionnels du développement présentaient l'état de la question à la fin des années 1970. Leur analyse partait d'un double constat: a) la "Participation populaire" dans le développement définie comme "l'implication (involvment) d'un nombre significatif de personnes dans des situations ou actions qui améliorent leur bien être" représenterait une stratégie radicalement différente de la conception fondée sur une théorie privilégiant l'initiative des agences de développement et le recours au capital, qui implique la passivité de la majorité de la population. La Participation populaire est conçue comme une condition et non plus comme une conséquence du processus de développement; b) cette conception, centrée sur les besoins fondamentaux des populations et sur leur propre capacité d'organisation, représenterait un tournant décisif mais récent vis à vis des théories et des pratiques de développement antérieures.

Plus de dix ans après l'article de Cohen et Uphoff, les débats actuels laissent penser que leur constat s'est largement vérifié. D'une part la philosophie du "modèle participatif' de développement rural s'est imposée auprès des catégories les plus diverses d'agents intervenant dans le développement. Les ONG, mais aussi les institutions internationales d'aide et de financement du développement, les services de coopération des principaux pays industrialisés, les universitaires, les publicistes tiennent pour évidence que les populations et, au premier chef, les agriculteurs doivent être associés à la définition des objectifs des projets et doivent participer à la mise en œuvre des moyens adaptés à ces objectifs. D'autre part, la très grande majorité des agents et des agences de développement se réfèrent à la Participation comme à une conception alternative récente et désormais incontournable, à la fois en tant que modèle intellectuel et en tant 
que modèle d'action. En bref, tout se passe comme si le "développement participatif' (terme générique recouvrant diverses appellations: développement à la base, décentralisé, communautaire, auto-centré, coopératif, par autopromotion paysanne etc.) était l'aboutissement d'un processus cumulatif des savoirs et des expériences sur le développement rural dont l'application permettrait d'attendre raisonnablement de meilleurs résultats que ceux enregistrés jusqu'ici.

3 Je voudrais non pas évaluer en tant que telle cette opinion (il y a, a priori, de bonnes raisons de penser qu'elle contient au moins une vérité de bon sens même si, comme pensée dominante, il lui arrive de charrier des éléments dogmatiques) mais la replacer dans l'histoire des idées et des pratiques du développement rural depuis la déjà lointaine époque où les administrations coloniales se préoccupèrent de formaliser leur action auprès des agriculteurs et des communautés rurales.

4 Nous en tirons, pour notre part, trois thèses qui éclairent d'un jour un peu différent la nature et ce que l'on peut attendre du programme présumé alternatif de développement fondé sur la participation: 1) Sur le plan de l'histoire des idées, il semble patent que la "participation des populations" a très tôt constitué la pierre angulaire des conceptions en matière de développement rural; elle constitue en réalité le modèle de référence des discours" développementistes" depuis la fin de la première guerre mondiale; 2) Sur le plan de l'histoire des organisations de développement, on peut observer de la même manière une très forte continuité dans le type de structure suscité par le secteur du développement en milieu paysan: dès la période de l'entre-deux-guerres, les administrations coloniales mettent en place et encouragent des structures inspirées par le modèle participatif : sociétés indigènes de prévoyance, mutuelles, coopératives, associations...; 3) Il est difficile d'opposer radicalement, d'une part les tentatives "anciennes" d'associer les paysans au développement, qui seraient caractérisés au mieux par une simple rhétorique participative, et, d'autre part, les pratiques récentes des structures contemporaines de développement "à la base" qui seraient capables de mettre en œuvre une participation effective. Il semble plutôt que nous ayons affaire à une configuration à la fois intellectuelle, culturelle et sociale à peu près constante, typique du dispositif institutionnel du développement tel qu'il s'est cristallisé à partir de la Première Guerre mondiale tant dans les métropoles que dans les colonies africaines d'alors, et tel qu'il s'est reproduit depuis sans grand changement.

5 Je présenterai surtout les éléments historiographiques qui permettent de soutenir ces thèses relatives à la récurrence du modèle participatif dans l'histoire institutionnelle du développement rural en Afrique de l'ouest depuis la colonisation. J'indiquerai cependant, en conclusion, quelques éléments théoriques qui permettent de comprendre en quoi cette lecture de l'histoire (que l'on pourrait qualifier à première vue de "révisionniste") peut se justifier par l'approche anthropologique du monde des "développeurs" et, réciproquement, doit encourager cette approche comme un complément indispensable à l'anthropologie des "développés".

L'entre-deux-guerres et le populisme colonial

6 On peut repérer précisément le moment où se diffuse de manière décisive le "modèle participatif de développement rural dans les administrations coloniales britannique et française : lorsque, passée la phase de conquête militaire, les tentatives d'implanter les entreprises prévues de colonisation échouèrent et lorsque l'expérience "en vraie grandeur", durant la première guerre mondiale, d'une économie administrée de type 
tributaire se révéla irréaliste (phillips, Henry, Pelleray, Sarraut). Après la guerre, l'administration coloniale française est de plus en plus fortement influencée par l'Indirect Rule britannique et l'association des paysans devient le maître-mot contre l'exploitation autoritaire et destructive de ressources prônée auparavant par le "Pacte colonial". Loin d'en être le simple prolongement, comme il est dit souvent, la politique de "mise en valeur" prônée par le ministre Sarraut en prend le contre-pied pour promouvoir "l'accroissement économique et le développement humain". Un extrait du "programme agricole de l'AOF pour 1921" élaboré par l'Inspecteur général de l'agriculture de l'AOF Yves Henry est suffisamment explicite quant à l'émergence précoce du modèle participatif (Henry 1922) (passages soulignés par nous J-P. C)

7 "Éduquer le paysan, lui donner les moyens de s'outiller, puis d'apporter progressivement à sa terre les améliorations foncières sans lesquelles tout programme ne serait qu'un vain mot, quel horizon nouveau ces nécessités ouvrent à la politique africaine et combien de questions inattendues elles posent aux pouvoirs publics !

8 On n'en retiendra ici que trois, les trois principales, et dans l'ordre où elles se présenteront dans la pratique.

9 La première, elle se posera demain. Comment réalisera-t-on le groupement des producteurs et quels moyens financiers mettra-t-on à leur disposition pour l'amélioration de l'outillage et de la culture? L'expérience répond: organisez la mutualité agricole, sous ses formes les plus diverses et particulièrement par le syndicat, la caisse de crédit, la coopérative de producteurs.

10 En A.O.F. ces associations trouveront une base excellente dans les Sociétés de Prévoyance dont quelques unes ont, au Sénégal, un actif important. Mais elles n'auront une existence assurée, elles ne rempliront leur office qu'à la double condition d'être vraiment des associations agricoles et de voir briser le cadre qui fait des sociétés de prévoyance actuelles des organes purement administratifs et qui les étouffe. Le paysan ne les comprendra, son initiative ne sera éveillée, il n'en tirera pleinement profit que si ces organes sont extrêmement souples et que si la tutelle administrative nécessaire au début se fait de plus en plus lâche, pour disparaître finalement et être remplacée par un simple pouvoir de contrôle."

11 Durant l'entre-deux-guerres, experts et administrateurs coloniaux, tant britanniques que français, formalisent une théorie cohérente de la politique agricole fondée sur la prise en compte des spécificités du "paysan noir" et l'impossibilité de tout autre alternative que la promotion de ce "paysannat" ou de la "voie paysanne" pour développer l'agriculture (phillips et Richards pour les colonies britanniques ; Delafosse, Labouret, Brévié, Delavignette, le juriste Lampué, l'agronome Adam notamment pour les colonies françaises). L'entreprise coloniale se veut explicitement rationnelle et scientifique, et fondée sur l'observation et l'utilisation d'un savoir organisé, fondé 1) sur le caractère incontournable de l'association des paysans aux projets coloniaux (versus la contrainte administrative) 2) la réhabilitation des savoir-faire et de la rationalité des pratiques paysannes; 3) l'appel aux sciences sociales, notamment aux anthropologues, sur le modèle britannique piloté par B. Malinowski ; 4) la méfiance à l'égard des effets dé structurants sur la paysannerie exercés tant par les entreprises commerciales européennes que par les nouvelles couches sociales africaines ("évolués" contestataires, "féodaux" enrichis etc.). Il est donc remarquable qu'en dépit du caractère intrinsèquement despotique du régime colonial, administrateurs et experts 
coloniaux formalisent une conception explicitement anti-capitaliste, agrarianiste et populiste du développement rural.

Qu'en est-il plus précisément des pratiques et des structures d'intervention mises en œuvre durant l'entre-deux-guerres? Dès la fin de la première guerre mondiale, ces conceptions se concrétisent en A.O.F. par les Sociétés Indigènes de Prévoyance (censées utiliser l'institution africaine traditionnelle des greniers de réserve collectifs) et les mutuelles de crédit et, dans les colonies britanniques, par les coopératives (avec création d'un corps de fonctionnaires coloniaux spécialement chargés de les soutenir) et la tolérance des Farmers'Unions. Les nouveaux "professionnels" du développement rural colonial s'inspirent des moyens mis en œuvre dans le secteur agricole métropolitain. Dans le cas français, les administrateurs civils (politiques et techniciens) reproduisent le modèle métropolitain du "Pacte social" mis en œuvre par la IIIème République: pour des raisons essentiellement politiques, l'État cherche à s'assurer l'alliance des couches paysannes (jusque là sous l'influence de l'aristocratie terrienne et de l'Église) en contrepartie d'une protection économique, d'une aide à l'organisation et d'un contrôle prudent de la modernisation (Marloie, 1986). La "politique du paysannat" prôné en Afrique est largement analogue à la "politique de Maintenance" du ministre Mélines en France, et l'organisation agricole créée en 1919 pour les agriculteurs métropolitains (offices, vulgarisation, coopératives, syndicats, mutuelles) inspire clairement le programme de l'Inspecteur général de l'Agriculture de l'AOF cité plus haut (Cerf et Lenoir 1987).

13 Un peu plus tard, les politiques interventionnistes mises en œuvre lors de la crise des années 1930 en Métropole est une source explicite d'inspiration pour le Gouvernement général de l'AOF. Pour Brévié, la mise en valeur agricole des colonies ne doit pas être recherchée dans une "doctrine coloniale spécifique" mais dans les tendances éprouvées dans les civilisations occidentales. Après 1936, le gouvernement du Front Populaire prolonge cette conception d'une "économie dirigée d'intérêt général aux colonies" associée a un "grand programme coopératif" (Mérat 1947). Les idées socialisantes et agrarianistes étaient d'ailleurs présentes chez les administrateurs coloniaux dès cette époque...

De la Seconde Guerre mondiale aux Indépendances : le triomphe du développement communautaire et coopératif

14 A partir de la seconde guerre mondiale, et jusqu'aux indépendances, triomphent le modèle du Community Development dans les colonies britanniques, celui du "relèvement rural" fondé sur l'animation rurale et les mouvements coopératifs et mutualistes dans les colonies françaises. Les institutions internationales, dont l'influence devient déterminante, reprennent en l'amplifiant le modèle du développement communautaire privilégié par les États-unis et la Grande Bretagne. Loin de constituer une rupture avec les idées coloniales de l'avant-guerre (comme on le dit souvent), ces modèles s'en inspirent directement (d'autant qu'ils émanent des mêmes cercles de hauts fonctionnaires ou d'experts coloniaux). On retrouve donc les mêmes principes, réactivés par la situation spécifique de l'après-guerre: le "développement communautaire" et "l'animation rurale" sont privilégiés comme modèle d'action non révolutionnaire dans le contexte de la guerre froide et de la montée des nationalismes.

On retrouve réaffirmées la conception "holistique" du développement rural (Delavignette, Gautier-Walter, Robequain, Mamadou Dia...), la rationalité et l'efficacité techniques de la "coutume agraire" indigène (Delavignette) ou du "système 
d'agriculture coutumier" (de Schlippé, qui est sans doute l'auteur qui a le plus précocement formalisé ce que l'on appelle aujourd'hui "the agricultural indigenous knowledge") et la rationalité économique des comportements paysans dans un contexte de système foncier communautaire et de transition des structures économiques (Binns de la FAO, et de Schlippé par exemple). On souligne le primat des "besoins normaux", des "felt needs" et de "l'équilibre de la cellule agraire", le scepticisme légitime des paysans à l'égard des interventions coloniales antérieures et leur défiance à l'égard des "actions extérieures" (Beckett, Staner, Delavignette, Sautter, de Schlippé, Assistance technique en AOF, Ancian, Staatz et Eicher...). Selon certains de ces auteurs, ce sont précisément la capacité d'adaptation et la rationalité des "paysannats" ouest-africains qui les engagent dans une évolution pervertie par le contexte économique et politique colonial. Dans ce contexte, la monétarisation et les droits fonciers traditionnels conduisent au gaspillage des ressources naturelles (forêt, fertilité des sols) et les structures d'interventions sont pénétrées par les enjeux politiques et les "ploutocraties" locales (de Schlippé, Harroy, Beckett, Robequain, Dia, Sautter, Binns...)

Toujours en continuité avec les principes identifiés dans l'entre-deux-guerres, se diffusent les idées de ce que l'on appelle aujourd'hui la "technologie appropriée" et la Recherche/Développement. La très grande majorité des auteurs insiste sur la nécessaire progressivité des interventions, à partir de la connaissance du "système agricole indigène", en excluant toute expérimentation hasardeuse ou risquée, et en faisant appel pressant aux sciences sociales, notamment à la sociologie, l'anthropologie et l'ethnologie. En tout état de cause une évolution rapide est jugée illusoire du fait des moyens d'action limités des services techniques coloniaux (Harroy, de Schlippé, Sautter, Beckett, Delavignette, Van Lier, Gautier-Walter...). Au passage, déjà, les "grands projets" impliquant des structures technocratiques et autoritaires dévoreurs de budgets sont égratignés (pélissier, à propos de l'office du Niger ; Beckett, à propos de l'aménagement de la Volta...).

17 Ainsi, à partir de la guerre, "développement communautaire", coopératives et mutuelles apparaissent les clés d'un développement rural à la fois techniquement efficace et politiquement légitime (Beckett, un des artisans de la recherche agro-économique en Afrique de l'Ouest britannique : "la politique et les idéologies sont aussi des facteurs d'organisation agricole"). Ici encore, se manifeste chez les professionnels du développement colonial de l'après-guerre l'influence des "techniques d'encadrement" prévalant à l'époque dans les métropoles ou dans les agricultures "développées": organismes de régulation du marché (Caisses de stabilisation, Marketing Boards), financement des infrastructures agricoles (Colonial Development and Welfare Acts, Fonds d'investissement pour le Développement Économique et Social) ainsi que des structures locales de développement prenant en compte les besoins non strictement économiques des agriculteurs (Community Development, animation rurale). Dans le cas français, la politique "d'encadrement rapproché" prônée pour les producteurs africains est analogue au foisonnement d'initiatives qui, dès la fin de la guerre, se multiplient pour favoriser la diffusion du progrès chez les agriculteurs français (Cerf et Lenoir, Bouée). Ici et là, sont réaffirmés les principes de la responsabilité de l'agriculteur, de l'initiative à la base et de l'importance du groupe... mais dans le cadre d'une forte mobilisation des services agricoles de l'État.

Le développement rural des années 1960 aux années 1970 : l'invention d'une tradition-repoussoir 

pratiques de développement rural après les indépendances est plutôt celle de l'échec de politiques productivistes et technocratiques, privilégiant des projets ambitieux et des structures de vulgarisation lourdes et contraignantes. C'est d'ailleurs ce diagnostic qui, au cours des années 1970, a justifié le qualificatif «d'alternatif» appliqué au modèle participatif. En réalité le modèle de développement rural prôné et mis en pratique dans la période suivant les Indépendances ne fut pas aussi éloigné de la norme « participationniste » des périodes précédentes, ni de la période qui suivit à partir des années 1970.

19 En premier lieu, s'il est vrai que les projets et politiques de développement des années 1960 promurent des objectifs ambitieux et technocratiques (en une période d'abondance croissante de l'aide extérieure, il faut le souligner), il n'en reste pas moins qu'ils promurent aussi, dans le cadre de ces projets et de ces politiques, les mêmes formes d'encadrement, de vulgarisation et d'animation, notamment en privilégiant le mouvement coopératif et l'animation communautaire (c'est l'époque du «socialisme africain », du "développement solidaire» et de la forte influence en Afrique francophone, du courant «Économie et Humanisme » de R.P. Lebret et F. Perroux au sein même des organes de planification).

En second lieu, par un curieux paradoxe, la "lourdeur" du dispositif de développement rural mis en place à cette époque (planification, opérations lourdes de développement sectoriel, politiques d'aménagement régional) est justifiée par ses promoteurs par le manque d'efficacité et les effets pervers des structures participatives mises en place dans les années 1950... mais en référence à l'objectif « participationniste » lui-même. En effet, le développement communautaire et le mouvement coopératif sont suspectés, à la fin des années 1950, de ne pouvoir jouer tout seuls le rôle qui leur est assigné. Sont invoqués : la lourdeur des structures de développement communautaire ou coopératif ; l'inefficacité des agents locaux qui ne peuvent assumer la polyvalence de ces structures, et qui, plus proches des élites locales que de la masse des producteurs, se révèlent paternalistes, élitistes et dirigistes; l'échec de ces structures quant à la production alimentaire de base; enfin, les effets technocratiques et inégalitaires sécrétés par ces structures... participatives (Holdcroft, Beckett, Delavignette, Robequain, Dia, Staatz et Eicher).

21 Ainsi, par une curieuse anticipation, les mêmes raisons, invoquées à la fin des années 1970 pour promouvoir un « développement par le bas », contre le développement " par le haut » des années 1960 et 1970, l'étaient déjà à la fin des années 1950 pour souligner les écueils organisationnels du développement communautaire et coopératif et pour promouvoir... une participation paysanne plus large. C'est bien dans ce sens qu'il faut comprendre les acteurs de la mise en oeuvre de la "planification ambitieuse " des années 1960 (multipliant cependant les structures de type coopératif...) et des « grands projets » des années 1970 (accouchant toutefois du « développement intégré » qui en partage le même esprit), dont on attend qu'ils assurent les pré-conditions d'un développement équitable.

Enfin, en troisième lieu, c'est davantage le contexte socio-politique local que la « philosophie » des projets de développement qui spécifie les années 60-70: contexte de restructuration politique, marqué par l'emprise de nouvelles couches locales dominantes sur les leviers de redistribution des ressources locales mobilisables depuis l'après-guerre par les institutions d'intervention économique (marketing boards, 
caisses de stabilisation, organismes de crédit et de commercialisation...). Pour l'essentiel, les grands projets sont financés par l'aide ou les financements extérieurs croissants et se superposent, sans les faire disparaître, aux structures d'encadrement très imprégnées de l'idée de participation. Les projets de développement des années 1960 et 1970 n'apparaissent donc pas aussi qualitativement différents des projets contemporains. Si les analystes contemporains les voient ainsi, ce n'est pas parce que la P.P. était alors moins affichée mais plutôt parce que, simultanément, elle était mise en œuvre par une "technostructure" censée protéger "par le haut" les structures participatives des effets pervers initiés "par le bas". C'est ce dernier trait qui est le seul retenu dans les critiques contemporaines du développement rural des années 1960 et au début des années 1970, alors que se fait jour une perte de confiance dans la capacité des États à impulser des actions de développement... et dans un contexte de raréfaction relative du financement extérieur.

Tout se passe donc comme si le développement rural des années 1960 et du début des années 1970 servait (et sert aujourd'hui encore) d'image-repoussoir au modèle participatif, au prix d'une réinterprétation a posteriori du passé, mais avec une gratification de taille: celle qui consiste, pour les promoteurs du développement participatif des années 1970, à pouvoir se réclamer d'un modèle "alternatif" opposé au modèle présumé "traditionnel" du développement "par le haut" des années 1960 et, par extension logique, de la période coloniale précédente.

Éléments pour une anthropologie des développeurs

Ce rapide survol historique permet de penser que, contrairement aux hypothèses de départ de Cohen et Uphoff et aux idées couramment admises, le modèle participatif de développement ne constitue nullement une conception alternative et récente de développement rural. En outre, ces conceptions ont depuis longtemps suscité des structures organisées, de formes semblables, censées les traduire dans la réalité. Il est historiquement inexact de tenir pour pure rhétorique le discours "associationniste" ou "participatif" tenu par les "développeurs" institutionnels depuis la première guerre mondiale. Cette critique "idéologique" du discours et des pratiques coloniales et post-coloniales n'est évidemment pas dénuée de pertinence, mais elle s'attache davantage à l'analyse des résultats plutôt que des causes. Elle attribue en outre une fonction explicative trop exclusive et univoque à une "sociologie de l'intérêt" extrêmement réductrice vis-à-vis des systèmes de représentations des "développeurs coloniaux" et de ceux qui leur ont succédé (la manière de concevoir ses intérêts est un construit social et culturel infiniment plus complexe que ce qu'évoque la notion "d'intérêts objectifs").

Ce faisant, elle n'explique pas certains éléments-clés de l'émergence et de la récurrence $\mathrm{du}$ modèle participatif dans l'histoire institutionnelle du développement rural, notamment : 1) pourquoi le choix de la P.P. comme modèle de référence à l'action de développement s'est imposé si précocement contre le modèle de la contrainte, qui pouvait aussi facilement se justifier, et se trouve souvent justifié "off record" de manière plus furtive, par les opérateurs contemporains, à partir des valeurs autoritaires et paternalistes; 2) pourquoi les organisations coloniales ou contemporaines de développement rural ont dépensé énergie et ressources pour identifier les causes de dysfonctionnement des structures participatives et pour tenter d'y remédier en les réformant constamment (et pas seulement pour des besoins de propagande). On a l'habitude de dire, par exemple, que les Sociétés Indigènes de 
Prévoyance, Sociétés de Prévoyance, Sociétés de Prévoyance Rénovées, Sociétés Mutuelles de Production Agricole, et autres Sociétés Mutuelles de Développement Rural qui se sont succédé en Afrique francophone n'étaient pas de véritables coopératives, notamment à cause du poids de l'administration ou des notables locaux. Mais c'est précisément ce problème qui inquiétait périodiquement les promoteurs (coloniaux) de ces structures et qui suscita leurs réaménagements successifs à partir d'analyse détaillées de leurs dysfonctionnements qui n'épargnaient pas les pratiques des responsables coloniaux locaux. Exactement de la même manière (et à partir des mêmes critiques), les auteurs contemporains parlent de la difficulté d'asseoir des structures participatives réelles; y compris lorsqu'il s'agit de structures initiées par des O.N.G.

L'apparente dissidence des tenants du « développement par le bas » qui s'est cristallisée depuis les années 1970 est donc en réalité un élément récurrent de l'histoire institutionnelle du développement. En conséquence, loin de pouvoir l'expliquer, la fonction critique sécrétée par les institutions de développement est elle-même un élément, à expliquer, de cette histoire.

Je voudrais, en guise de conclusion, esquisser une grille d'analyse qui permette de lever le paradoxe auquel aboutit cette reconstitution historique. Ce paradoxe peut être formulé de deux manières: d'une part, la prégnance dans les institutions de développement du modèle participatif de développement rural de l'époque coloniale à aujourd'hui, indépendamment du régime politique réel et en dépit des affinités logiques de ce modèle avec les conceptions démocratiques du pouvoir et de l'autorité ; d'autre part, la permanence d'une attitude auto-critique des agents du développement alors que l'on attribue plutôt les faibles performances des structures d'intervention à leur tendance à se reproduire pour elles-mêmes. Il me semble que quelques remarques théoriques peuvent aider à expliquer ce double paradoxe.

On peut d'abord envisager le dispositif spatio-temporel de développement comme une institution sociale à part entière, contribuant à réguler les rapports entre les groupes locaux, les sociétés paysannes, les sociétés globales et les États (chacun de ces niveaux n'étant d'ailleurs pas homogène). Si nous utilisons la méthodologie weberienne de l'idéal-type, l'histoire institutionnelle du développement semble pouvoir être caractérisée par la combinaison de deux types de légitimité, avec leurs systèmes de valeurs, d'autorité et d'action spécifiques.

29 Le premier est le type bureaucratique de légitimité et d'autorité (correspondant à l'organisation légale-rationnelle que Weber oppose aux types traditionnel et charismatique). Développer, c'est d'abord opérer un transfert du type "rationnel » d'organisation sociale dans des milieux sociaux où d'autres types d'autorité légitimes sont prééminents. Ce transfert consiste, d'une part, à mettre en œuvre des organisations bureaucratiques spécialisées dans la conception et l'administration du " changement provoqué », et, d'autre part, à inciter les groupes sociaux bénéficiaires des interventions à s'organiser eux-mêmes selon des variantes de ce modèle rationnel (groupements, coopératives, conseils etc.). En outre, conformément à ce modèle, sont mises en place des procédures de contrôle et d'évaluation critique des résultats de ces organisations bureaucratiques.

Un deuxième élément caractéristique de l'histoire institutionnelle du développement est sa composante "populiste ». Dans le même sens que les observations précédentes, mais pour une période plus récente, un certain nombre d'auteurs (Kitching, Robertson, Richards, Olivier de Sardan...) ont bien mis en évidence la disposition des développeurs 
à reconnaître la nécessité de s'appuyer sur les ressources de « la base », tant sur le plan de l'efficacité qu'à celui de la légitimité. Cette disposition s'est cristallisée en fait très précocement en une "culture du développement ", d'abord à l'usage des populations « arriérées » ou démunies des métropoles, puis dans les dispositifs de " mise en valeur » et de développement des pays colonies. La culture populiste du développement est à l'origine d'une représentation stéréotypée du milieu rural africain (invariablement communautaire, solidaire et égalitariste) qui se perpétue depuis que les agents du développement colonial ont cru voir dans la petite agriculture marchande qui se diffusa dans l'entre-deux-guerres l'héritière $d u$ " vieux fonds paysan africain ».

31 Mais la combinaison de ces deux composantes dans l'histoire et la culture institutionnelles du développement est porteuse de contradictions permanentes. Elle prétend en effet concilier les vertus de l'efficacité formelle et dépersonnalisée de l'organisation bureaucratique et les vertus de l'organisation communautaire populaire. Or de nombreuses études illustrent comment la combinaison de ces éléments contradictoires que sont l'idéal bureaucratique et l'idéal communautaire aboutit simultanément à un renforcement du formalisme bureaucratique, d'une part, et, d'autre part, au renforcement de micro-réseaux particularistes assimilé à l'expansion du clientélisme et de la corruption. Les insuffisances de fonctionnement des organisations locales sont attribuées au particularisme des sociétés et cultures locales et corrigées par davantage de règles et de contrôle; les pratiques pragmatiques pour les adapter sont considérées comme manifestation d'inaptitude et de corruption; néanmoins les caractéristiques communautaires des collectivités paysannes sont sollicitées pour assurer aux organisations paysannes cohérence, représentativité et durée. A partir de là se déroule un processus de "malentendu productif" et d'ajustement sans fin entre les "stratégies paysannes" (révélant bien évidemment l'hétérogénéité sociale et économique du milieu paysan) et les stratégies des opérateurs de développement (révélant tout aussi bien le caractère politique et contraignant de leur action). La lutte contre les dysfonctionnements du "populisme bureaucratique" alimente la croissance de la bureaucratie et nourrit les stratégies paysannes de "détournement" des objectifs bureaucratiques.

Les administrateurs, experts et techniciens coloniaux n'étaient pas moins au fait de ces problèmes que les critiques contemporains du style bureaucratique de développement. La culture du développement s'est ainsi cristallisée comme un dispositif cognitif soumis en permanence à l'existence d'effets non attendus ou contre-productifs entravant son instrumentalisation. $\mathrm{n}$ s'en suit que la culture du développement s'est professionnalisée principalement sur la base d'une codification de ces effets que la fonction critique inhérente à l'exercice de la rationalité bureaucratique ramène sans cesse à la conscience des développeurs.

33 Ainsi, une fois "inventée" l'image populiste de la paysannerie africaine (au sens où cette image est une construction culturelle historiquement située), l'écart entre les effets attendus de l'intervention de développement et les résultats observables peut être interprété de différentes manières. Cet écart - invariablement assimilé à des effets pervers contingents - peut être imputé: a) soit aux défauts de l'organisation bureaucratique lorsqu'elle se transforme en une technocratie; b) soit à l'intervention perverse d'intérêts émanant de couches sociales dominantes non-paysannes, par exemple les politiciens, les administrateurs ou les commerçants ou, à l'échelle internationale, les représentants des firmes et des grands pays industriels; c) soit 
encore (version "misérabiliste") à l'état de décomposition du milieu paysan lui-même qui, sous l'effet de la domination exogène, perd ses qualités "populaires" et se trouve gagné par l'individualisme, l'inégalité ou tout simplement l'anomie engendrée par une grande détresse matérielle et morale.

Or on peut observer que l'ensemble de ces arguments est régulièrement invoqué depuis la fin de la première guerre mondiale dans l'histoire institutionnelle du développement rural. Tout se passe comme si leur combinaison, coexistence ou opposition au sein de la culture des développeurs suscitait la redécouverte constante du problème de la Participation populaire et provoquait en permanence la réactivation de la fonction critique de la composante bureaucratique de la culture du développement tout en réactualisant les valeurs populistes, réamorçant ainsi la production d'effets d'ajustement des rapports sociaux entre Développeurs et Développés, entre les différentes couches rurales et entre les diverses composantes organisationnelles et hiérarchiques du dispositif de développement lui-même. Ce sont ces processus d'ajustement - socialement "normaux" - que la culture du développement codifie comme des processus pervers, ménageant ainsi les conditions d'un nouveau cycle d'ajustement. Ce processus "idéal-typique" fait de la fonction critique inhérente à la composante bureaucratique de l'institution du développement un élément-clé de sa reproduction, par la "réinvention" constante et régulière du développement participatif comme parangon et modèle indépassable du développement. légitime et efficace.

Ce processus explique également que, dans l'histoire institutionnelle du développement, la prégnance $\mathrm{du}$ modèle de développement participatif est relativement indépendante des pratiques dans la sphère proprement politique. Sa réactualisation s'accommode de (ou peut être générée par) les idéologies ou régimes politiques les plus divers, voire même opposés. Le "populisme colonial" a trouvé sa "niche idéologique" aussi bien dans des phases de pur despotisme (comme dans l'entre deux-guerres où le mode dominant de gouvernement est celui du paternalisme autoritaire), de réformisme (après la seconde guerre et pour faire pièce à la fois à la montée des revendications nationales et aux pressions internationales) ou de partage du pouvoir avec les partis nationalistes dans les années 1950 (période durant laquelle "s'indigénise" la culture bureaucratique et populiste du développement). De même, après les Indépendances et le "transfert institutionnel" qui s'en suit, le développement communautaire et le coopérativisme fleurissent aussi bien dans les pays africains à régime dictatoriaux que dans ceux qui ménagent des formes démocratiques. A défaut de garantie démocratique dans la sphère proprement politique, la conformité au modèle participatif a longtemps suffit aux agences d'aide au développement rural pour accorder leur soutien aux régimes en place...

Il nous semble que prendre la mesure de cette histoire ambiguë, loin d'être démobilisateur du point de vue de l'action et, disons-le, du point de vue de la philosophie politique qui sous-tend le modèle participatif, devrait nous engager à ne jamais séparer l'anthropologie du développement.. de l'anthropologie des développeurs. 


\section{BIBLIOGRAPHIE}

Adam J., 1937 - "De l'écologie agricole à l'écologie coloniale", L'agronomie coloniale, nº 237 (sept. 1937).

Ancian G., 1956 - La modernisation du paysannat dans les territoires d'outre-mer, La Documentation Française, Notes et Études Documentaires, $\mathrm{n}^{\circ} 2129$.

Anonyme, 1959 - "Une forme de vulgarisation agricole d'assistance technique en AOF", Centre CCTA/OAA pour le développement de la vulgarisation agricole, Ibadan.

Anouma R., 1977- "Les sociétés indigènes de prévoyance, la Caisse Centrale de Crédit Agricole Mutuel : organisation, fonctionnement et rôle en Côte d'Ivoire jusqu'à la veille de la 20 guerre mondiale", Annales de l'Université d'Abidjan, série 1., t V.

Bare F., 1987 - "Pour une anthropologie du développement économique", Études Rurales, 105-106 : 267298.

Bates R.H., 1983 - Essays on the political economy of rural Africa, University of California Press.

Beckett W.H., 1953 - "Afrique occidentale britannique" in INCIDI 1953.

Beckett W.H., 1953 - "Économie rurale. Rapport général" in INCIDI 1953.

Binos Sir Bernard, 1953 - "Introductory Paper by the Organization of the United Nations", in INCIDI 1953.

Bosc P.M., Calkins P., Yung J.M., 1990 - Développement et recherche agricole dans les pays sahéliens et soudaniens d'Afrique, DSA-CIRAD, Montpellier.

Brasseur G., 1957 - L'A.O.F., IFAN-Dakar, Initiations africaines.

Brevie (Gouverneur Général), 1936 - Trois études, Gorée, Imprimerie du Gouvernement.

Caty R., 1936 - "L'amélioration des plantes de culture indigène aux colonies", L'agronomie coloniale, $\mathrm{n}^{\circ} 218$ (février 1936) et $\mathrm{n}^{\circ} 219$ (mars 1936).

Cerf M. et Lenoir D., 1987 - Le développement agricole en France, Paris, P.U.F., coll. Que sais-je ?

Cernea M.M., 1985 - Putting People First : Sociologial Variables in Rural Development, New-York, Oxford university Press.

Chambers R., 1983 - Rural Development : putting the last first, Longman, London (trad. fr. : Développement rural. La pauvreté cachée, Pais, C.T.A.-Karthala).

Cohen J.M., Culagovski G.A., Uphoff N.T., Wolf D.L., 1978 - Participation at the locallevel : a working bibliography, Cornel University.

Cohen J.M., Uphoff N.T., 1980- "Participation's Place in Rural Development : Seeking Clarity through Specificity", World Development, $8: 213-235$.

Delafosse M., 1927 - Les nègres, Paris, F. Rieder.

Delavignette R., 1931, Les paysans noirs, Paris, Stock.

Delavignette R., 1946 - Service africain, Paris, Gallimard.

Delavignette R., 1953 - "Politique et droits agraires. Rapport général" in INCIDI 1953. 
Dia Mamadou, 1952 - Contribution à l'étude du mouvement coopératif en Afrique noire, Paris, Présence Africaine, $3^{\circ}$ édition.

Dumont L., 1985 - "Identités collectives et idéologie universaliste : leur interaction de fait", Critique, XLI, n 456 : 506-518.

Eicher C.K. et Staatz J.M. ed., 1984 - Agricultural Development in the Third World Baltimore and London, The Johns Hopkins University Press.

Empire colonial français (L'), 1930 - Paris, Plon.

F.A.O., 1959 - Bien-être rural. Développement de la vulgarisation agricole. Conférence d'Ibaban 1959. Communications :- "Une forme de vulgarisation agricole d'assistance technique en A.O.F." - "Les rapports entre la vulgarisation agricole et la formation générale" (M. Gaudy).- "Note sur la vulgarisation agricole au Dahomey".

Foucault M., 1971 - L'ordre du discours, Paris, Gallimard.

Gautier-Walter A., 1951 - Afrique noire terre inconnue. La croisière noire de la santé, préface de L.S. Senghor, Paris, Frédéric Chambriand.

Gayet G., 1953 - "L'Office du Niger", in INCIDI 1953.

Gentil D., 1984 - Les mouvements coopératifs dans l'Afrique de l'Ouest "francophone", Thèse d'État, EHESS, 2 tomes.

Groupe de travail Coopération Française, 1989 - Les interventions en milieu rural. Principes et approches méthodologiques, M.C.D.

Harroy J.P., 1944 - Afrique, terre qui meurt. La dégradation des sols africains sous l'influence de la colonisation, Bruxelles, Marcel Hayez - Office international de Librairie.

Henry Y., 1922 - Le programme agricole. Exposé du programme. Campagne 1921, Gouvernement général de l'AOF, Paris Larose.

Holdcroft L.E., 1984 - "The Rise and Fall of Community Development", 1950-1965", in Eicher and Staatz 1984.

Houee P., 1989 - Les politiques de développement rural. Des années de croissance aux temps d'incertitude, Paris, INRA- Economica.

Hulme D. and Turner M., 1990 - Sociology and Development. Theories, Politics and Pratices, Harvester Wheatsheaf, New York.

Hyden G., 1983 - No shortcuts to progress. African development management in perspective, Berkeley, University of California Press.

Institut International des Civilisation Différentes (INCIDI), 1953 - Programmes et plans de relèvement rural en pays tropicaux et sub-tropicaux, C.R. de la $28^{\circ}$ session tenue à La Haye les 7, 8, 9 et 10 septembre 1953, Bruxelles.

Kitching G, 1982- Development and Underdevelopment in Historical Perspective. Populism, Nationalism and Industrialisation, London and New York, Methuen.

Labouret H., 1928 - "Le coton et l'indigène", Africa, 1, 3 : 320-337.

Labouret H., 1941 - Paysans d'Afrique noire, Paris, Gallimard

Labouret H., 1952 - Colonisation, colonialisme, décolonisation, Paris, Larose. 
Lachenmann G. et al., 1990 - Organisations paysannes au Sénégal : comment renforcer les structures intermédiaires dans le processus de transformation socio-économique, Institut Allemand de Développement, Berlin.

Lavigne-Delville P., 1990 - Groupements villageois et processus de transition, multigr.

Laville P., 1972 - Associations rurales et socialisme contractuel en Afrique Noire, Paris, Cujas, 1972.

Lele U., 1975 - The Design of Rural Development lessons from Africa, The Johns Hopkins University Press, Baltimore and London.

Lele U., 1984 - "Rural Africa : Modernization, Equity, and Long-Term", in Eicher and Staatz 1984.

Long N., 1984 - "Creating space for change. A perspective on the sociology of development", Sociologia Ruralis, XXIV - 3/4.

Marloie M., 1986 - "Les changements dans la régulation du marché des céréales en France depuis la fin du XIXe siècle", Études du CIHEAM-IAM, déc. : 217-230.

Meister A., 1977 - La participation pour le développement, Paris, Les éditions ouvrières.

Merat L., 1947- Fictions... et réalités coloniales, Paris, Recueil Sirey

Mercoiret M.R., 1990 - L'émergence des dynamiques locales. Une réponse au désengagement de l'État. Séminaire Club du Sahel-CIRAD, Montpellier.

Ministère de la Coopération, 1976, Réflexions nouvelles sur le Développement Rural, Études et Documents $\mathrm{n}^{\circ} 25$.

Ministère de la Coopération, 1989 - Les interventions en milieu rural. Principes et approches méthodologiques, Paris.

Morawetz D., 1977 - Vingt-cinq années de développement économique : 1950-1975, Paris, Economica-Banque Mondiale.

Olivier de Sardan J-P., 1990 - Populisme développementaliste et populisme en sciences sociales : idéologie, action, connaissance, Cahiers d'Études Africaines, XXX (4), 120 : 475-492.

Ouedraogo A.P., 1990 - "Le double langage du développement. La formation des jeunes agriculteurs au Burkina Faso", Cahiers d'économie et de sociologie rurale, $\mathrm{n}^{\circ} 14$ : 74-94.

Passeron J.C., 1989 in C. Grignon et J.C. Passeron - Le savant et le populaire, Paris, Gallimard. Le Seuil.

Pelissier P., 1953 - "Afrique occidentale française" in INCIDI 1953.

Pelleray E., 1923 - L'Afrique occidentale française. Le milieu, l'organisation, la mise en valeur. Le Togo. Edit. Notre Domaine Colonial, Paris.

Phillips A., 1989 - The Enigma of Colonialism, British Policy in West Africa, Londres, James Currey Indiana University Press.

Richards P., 1985 - Indigenous Agricultural Revolution, London, Hutchinson.

Robequain Ch., 1949 - Les richesses de la France d'Outre-Mer, Paris, Payot.

Robertson A.F., 1984 - People and The State. An Anthropology of Planned Development, Cambridge University Press.

Rolland L. et Lampue P., 1940 - Précis de Législation coloniale, Paris, Dalloz.

Ruopp P., ed., 1953 - Approches to Community Development, Bruxelles, Uitgeversmaatschpappij A. Manteau N.V. 
Sarraut A., 1923 - La mise en valeur des colonies françaises, Paris, Payot

Sautter G., 1953 - "Afrique équatoriale française", in INCIDI 1953.

Schlippe D. de, 1953 - "Le relèvement rural en fonction de la coutume agricole. L'expérience du Soudan anglo-égyptien", in INCIDI 1953.

Schmitz J., 1991 - "Les sciences et les techniques appliquées au développement et l'anthropologie", Bulletin de l'A.P.A.D. (Association euro-africaine pour l'anthropologie du changement social et du développement), $\mathrm{n}^{\circ} 2: 9-15$.

Sociologia Ruralis, 1988 - Aïd and Dévelopment, $n^{\circ}$ spécial, XXVIII - 2/3.

Soest J., Van, 1978 - The Start of International Development Cooperation in the United Nations 19451952, Yssen, Van Gorcum.

Spittler G., 1984 - "Peasants, the administration and rural development", Sociologia Ruralis, 24 : 7-9.

Spittler G., s.d. - L'administration coloniale en Afrique Occidentale Française (AOF) : despotisme et bureaucratie, multigr.

Staaz J.M. et Eicher C.K., 1984 - "Agricultural Development Ideas in Historical Perspective", in Eicher and Staatz 1984.

Staner P., 1953 - "Le développement rural au Congo Belge et au Rwanda-Urundi", in INCIDI 1953.

Teulings A.W.M., 1973 - "Modèles de croissance et de développement des organisations", Revue française de sociologie, XIV : 352-370.

Van Lier R.A., 1953 - "Vie sociale rurale. Rapport général" in INCIDI 1953.

Weber M., 1947 - The Theory of Social and Economic organization, ed. with an intr. of T. Parsons, The Free Press, New-York.

\section{AUTEUR}

JEAN-PIERRE CHAUVEAU

ORSTOM, Montpellier 